\title{
Empirical Study of the Telecommunication Operation Performance Evaluation of Regional China
}

\author{
Qu Yi ${ }^{1,2}$, Zhong Shen ${ }^{3}$, Zhang Dehua ${ }^{3}$ \\ (1 School of Economics in Harbin University of Commerce, Heilongjiang, Harbin, 150028) \\ (2 Department of Finance in Harbin Vocational College of Science and Technology, Heilongjiang, Harbin, 150300) \\ (3 School of Finance in Harbin University of Commerce, Heilongjiang, Harbin, 150028)
}

\begin{abstract}
Based on the total assets and cost of main operations as input variables, the main business revenue and profit as the output variable in this paper, we build the operational performance evaluation model of China's telecom industry, based on DEA-CCR, and use the relevant data to measure the operational performance of different regions. The empirical results show that: the performance level of the telecom operators in China is relatively high and is not gathered, there is further room for improving telecom industry.
\end{abstract}

Keywords-DEA-CCR; telecom industry; operational performance

\section{MODEl CONSTRUCTION}

Data Envelopment Analysis (DEA) is a method for evaluating the relative efficiency of the same type of multi index input and multi index output, as well as a powerful tool for multi-input multi-output function. In this paper, the DEA method is used to evaluate the performance of telecom industry in China.

Set up a total regions of $n \quad(n=30$, excluding Tibet because of data problems), known as an evaluation unit of n (n=30). $D M U_{j}(j=1,2, \cdots, 30)$, each evaluation unit has $m(m=2)$ kinds of inputs, respectively, the total assets and the main business cost and has $\mathrm{p}(\mathrm{p}=2)$ kinds of outputs, respectively, the main business revenue and total profit, so that there is an evaluation system of $\mathrm{n}$ unit for multi-index inputs and multi-index outputs ,as shown in Fig. I[1-5].

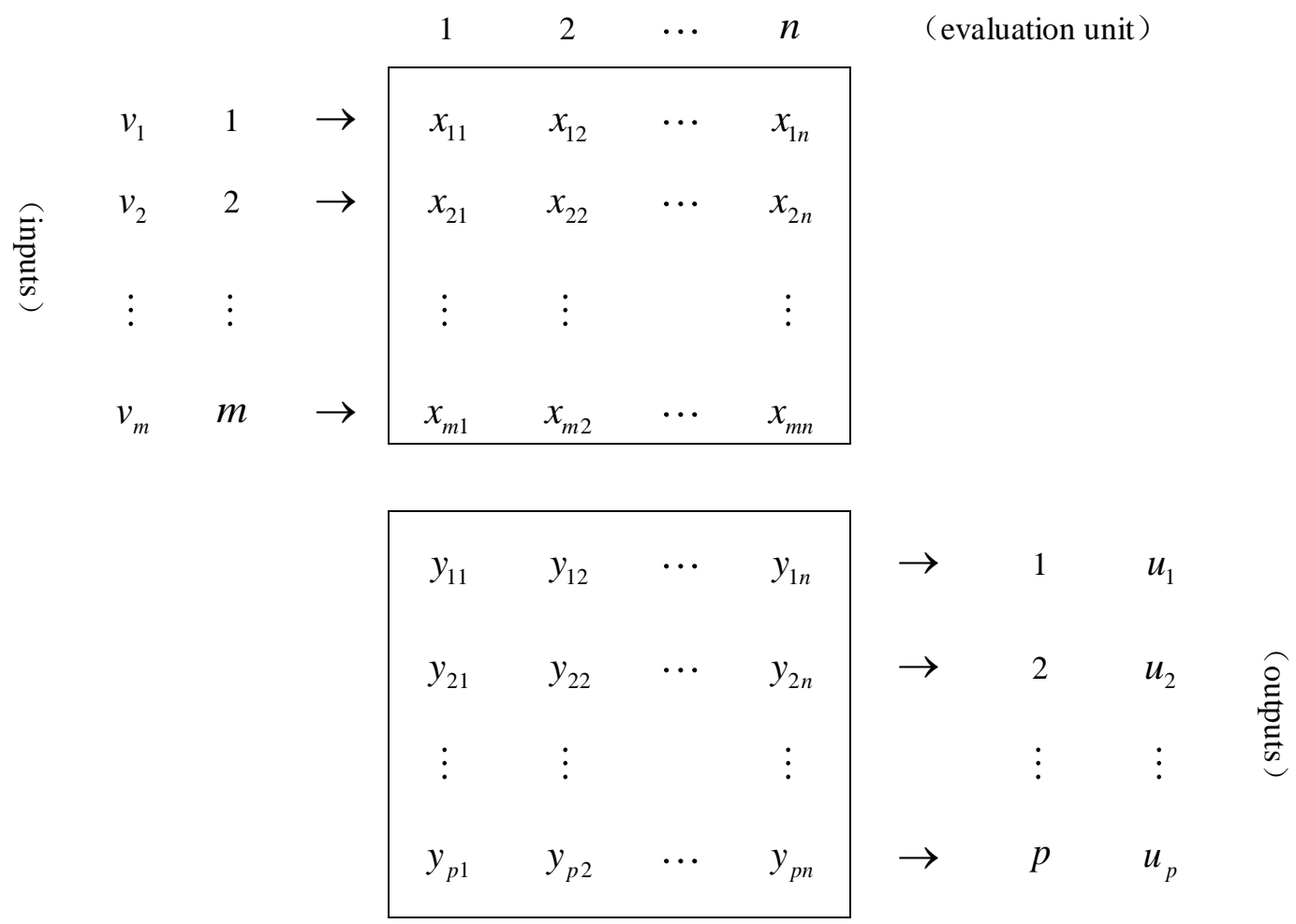

Figure 1. DEA evaluation system of telecom operators in China 
In the figure, $x_{i j}$ means the $\mathrm{i}(\mathrm{i}=1,2)$ kinds of evaluation of input indicators in $D M U_{j}$ evaluation unit, the model is the total assets and the main business cost $\left(x_{i j} \succ 0\right)$.

$y_{r j}$ means the $\mathrm{r}(\mathrm{r}=1,2)$ kings of evaluation of output indicators in $D M U_{j}$ evaluation unit, the model is the main business income and total profit $\left(x_{i j} \succ 0\right)$.

$v^{v \text { means the }} i(i=1,2)$ kinds of weight coefficient of input index $\left(v_{i} \geq 0\right.$ ).

$u_{r}$ means the $r(r=1,2)$ kinds of weight coefficient of output indicator $\left(u_{r} \geq 0\right.$ ).

$x_{i j}$ and $y_{r j}$ is the components of $x_{j}=\left(x_{1 j}, x_{2 j}, \cdots x_{m j}\right)^{T}$ and $y_{j}=\left(y_{1 j}, y_{2 j}, \cdots y_{q j}\right)^{T}$.The index data of this paper are derived from the "2012 China's third industry statistics yearbook".

In the evaluation system shown in Figure 1, the weight coefficient vector of the input index and output index in the model is $v=\left(v_{1}, v_{2}\right)^{T}$ and $u=\left(u_{1}, u_{2}\right)^{T}$ respectively.

For evaluation unit, the evaluation index is

$$
h_{j}=\frac{\sum_{r=1}^{p} u_{r} y_{r j_{0}}}{\sum_{i=1}^{m} v_{i} x_{i j_{0}}} \quad(j=1,2, \cdots, 30)
$$

By this definition, first we can always appropriately select $\mathrm{u}$ and $\mathrm{v}$ and make $h_{j} \leq 1$. Second, roughly speaking, for the evaluation unit of $D M U_{j_{0}}$, the greater the $h_{j_{0}}$, the greater the use of relatively small input to get relatively more output [6-11].

To evaluate the relative effectiveness of the $h_{j_{0}}$

evaluation unit, that is, if we want to understand this $D M U_{j_{0}}$ in $D M U$ with $n(n=30)$ is whether "optimal" or not, we shall establish CCR model evaluation system.

In the DEA-CCR evaluation model of Chinese telecom industry operators, to set up the input vector and output vector of the $j_{0}$ evaluation unit respectively as $x_{0}=\left(x_{1 j_{0}}, x_{2 j}\right)^{T}$ and $y_{0}=\left(y_{1 j_{0}}, y_{2 j_{0}}\right)^{T}$, and the operational performance index as $h_{0}=h_{j_{0}}$. Under the condition of the operating performance evaluation index $h_{j} \leq 1 \quad(j=1,2, \cdots, 30)$, to select the optimal weight coefficient $\mathrm{u}$ and $\mathrm{v}$, and make $h_{0}$ reaches the maximum and establish optimization DEA-CCR model [12-15].

$$
\left\{\begin{array}{l}
\max h_{0}=\frac{\sum_{r=1}^{p} u_{r} y_{r j_{0}}}{\sum_{i=1}^{m} v_{i} x_{i j_{0}}} \\
\text { s.t. } \quad \frac{\sum_{r=1}^{p} u_{r} y_{r j_{0}}}{\sum_{i=1}^{m} v_{i} x_{i j_{0}}} \leq 1 \quad(j=1,2, \cdots, 30) \\
v_{i} \geq 0 \quad(i=1,2) \\
u_{r} \geq 0 \quad(r=1,2)
\end{array}\right.
$$

\section{EMPIRICAL ANALYSIS}

\section{A. Tobit model description}

According to the above construction of China's regional telecom industry performance DEA-CCR evaluation model, can be obtained the corresponding operational performance measure values, specifically see tab. I.

TABLE I OPERATIONAL PERFORMANCE MEASURES OF THE TELECOM INDUSTRY IN CHINA (1)

\begin{tabular}{ccc}
\hline DMU & Operational performance & Ranking \\
\hline Beijing & 0.9071 & 21 \\
Tianjin & 0.8559 & 28 \\
Hebei & 0.9314 & 19 \\
Shanxi & 0.9334 & 17 \\
Inner Mongolia & 0.9351 & 16 \\
Liaoning & 0.9528 & 11 \\
Jilin & 0.8465 & 29 \\
Heilongjiang & 0.8919 & 24 \\
Shanghai & 0.9618 & 9 \\
Jiangsu & 1 & 1 \\
\hline
\end{tabular}

TABLE II Operational PERFORMANCE measures of the telecom industry in China (2)

\begin{tabular}{ccc}
\hline DMU & Operational performance & Ranking \\
\hline Zhejiang & 0.9665 & 7 \\
Anhui & 0.9418 & 13 \\
Fujian & 0.9372 & 14 \\
Jiangxi & 0.9317 & 18 \\
Shandong & 0.9583 & 10 \\
Henan & 0.8643 & 27 \\
Hubei & 0.9309 & 20 \\
Hunan & 0.9652 & 8 \\
Guangdong & 1 & 1 \\
\hline
\end{tabular}


Guangxi

0.9825

6

TABLE III Operational performance MEASURES of the telecom industry in China (3)

\begin{tabular}{ccc}
\hline DMU & Operational performance & Ranking \\
\hline Hainan & 0.8782 & 26 \\
Chongqing & 0.986 & 5 \\
Sichuan & 0.9421 & 12 \\
Guizhou & 1 & 1 \\
Yunnan & 0.9356 & 15 \\
Shanxi & 0.9961 & 4 \\
Gansu & 0.8932 & 23 \\
Qinghai & 0.7559 & 30 \\
Ningxia & 0.8974 & 22 \\
Xinjiang & 0.8881 & 25 \\
\hline
\end{tabular}

Through the above three tables, we can see that the different regions of China telecom industry operating performance is relatively good, 30 regions with an average of 0.9289 , the lowest value of 0.7559 in Qinghai, and the rest of the operating performance values are more than 0.8 . 0.9-1 of inter have 18 regions, there are eight regions between 0.8-0.9. Among them, Jiangsu, Guangdong and Guizhou, the three best performing regional telecom industry operators, are 1; Telecom industry operator performance of Qinghai, Jilin and Tianjin are the last three, respectively $0.7559,0.8465,0.8559$. Shanxi, Chongqing, Guangxi, Zhejiang, Hunan, Shanghai and Shandong are the regions at the forefront. Liaoning, Sichuan, Anhui, Fujian, Yunnan, Inner Mongolia, Shanxi, Jiangxi, Hebei and Hubei are in the middle region. Beijing, Ningxia, Gansu, Heilongjiang, Xinjiang, Hainan and Henan are in relatively backward areas.

\section{CONCLUSIONS}

By constructing the performance evaluation model of China's telecom industry in China based on DEA-CCR, we can get the following conclusions.

First, the overall level of operational performance of China's telecommunications industry is relatively high. In the 30 regions, only Qinghai's telecom industry performance below 0.8 is 0.7559 . Overall average of 0.9289 , there are three areas of relative performance to 1 . Thus, the overall development of China's telecommunications industry is good [16].

Second, there is no agglomeration state of the operational performance of the telecom industry [17]. Through the empirical results, we can see that there are some areas in the east, middle and west of the telecom industry with high performance, but also some areas of low performance. The top of the rankings does not drive the development of the telecom industry in the surrounding areas, and there is no obvious relationship between the level of economic development and the operation performance of the telecom industry. So it can be said that there is no obvious spillover effect on the operation performance of the telecom industry [18].

Third, the region's telecom industry has a further improvement of the space. In the 30 regions of China, only three regions, the relative performance of the telecom industry operation level is 1 , and the rest are to achieve the relative optimal state, there is 1 region below 0.8 . There are 18 regions between $0.9-1,8$ regions between $0.8-0.9$. Therefore, the regional telecom industry can also be further promoted, and the spillover effects of the telecom operators in the surrounding areas can also be utilized reasonably [19].

\section{ACKNOWLEDGEMENT}

This project is supported by the National Social Science Fund Project "Transfer of Financial Perspective Adjustment of China's Rural Financial System"(13BJY087).

\section{REFERENCE}

[1] Na Baoguo, Liang Jingguo. Based on fuzzy grey comprehensive evaluation method in China telecom industry international competitiveness evaluation [J]. Modern Management Science, 2008 (4): 32-33

[2] Zheng Shilin. Market competition or property rights reform has provided the telecom industry performance $[\mathrm{J}]$. World Economy, 2010 (6): 118-139

[3] Zhang Chao, Zhang Quan, Zhang Hong. Reform process and efficiency evaluation of China's telecom industry [J]. Statistics and Information Forum, 2012 (7): 86-91

[4] Wen Pingchuan, Luo Xianbo. Evaluation of the competitiveness of different regions in China based on grey system theory [ $\mathrm{J}]$. Modern Management Science 2012 (4): 46-49

[5] Liang Bing Development and financing survey report of Chinese SME [J] Financial Research, 2004 (5): 120-138

[6] Lin Yifu, Li Yongjun. Development of small and medium financial structure and SME financing [J]. Economic Research, 2001 (1): 10 18 ,

[7] BAAS SCHROOTEN., Relationship banking and SEMs: A theoretical analysis [J]. Discussion Paper German Institute of Economic Research, Berlin, 2005

[8] Wu Yechun, Wang Cheng. Empirical research on the growth factor model of SMEs [J]. Special Zone Economy, 2007 (6): 268-269

[9] Lin Wenqiong, Zhang Wenqi, Zheng Jinghui, Xie Shuichang. The empirical study of social capital in rural small and medium enterprises in the informal credit availability [J] Technical Economic, 2009 (5): 53-58

[10] Wan Qian, Liu Li. Regional financial environment and financing behavior of SMEs [J]. Finance Forum, 2010 (10): 73-80

[11] Zhang Jiangang, Wang Shanshan, Wang Hongqi. Research on the development strategy of Science and Technology Park of universities in Heilongjiang [J].Technical Economy, 2004 (1): 11 14

[12] Patton M. P, Kostov, S, McErlean, and J Moss. Assessing The Influence of Direct Payments On The Rental Value Of Agricultural Land [J]. Food Policy, 2008, Vol. 33 (5): 397-405.

[13] Kirwan, B. The Incidence of U.S. Agricultural Subsidies on Farmland Rental Rates [J]. Journal of Political Economy, 2009 (117): 138-164

[14] Zhang Dehua, Zhou Huiqiu and Lou Sha. The problems and the countermeasures in food production in Heilongiang [J]. Research of Agricultural Modernization. 2012(4):411-414.

[15] Lou Sha, Liu Huiping, Zhang Dehua. Comparative study on grain production and farmers' income coordination in Heilongjiang [J] Research of Agricultural Modernization. 2013, Vol.34 (6):654-658

[16] Patton M. P, Kostov, S, McErlean, and J Moss. Assessing The Influence of Direct Payments On The Rental Value Of Agricultural Land [J]. Food Policy. 2008, Vol. 33(5): 397-405.

[17] Zhou Qiren. Property and Institutional Change [M]. Beijing University Press, 2004.

[18] Zhang Dehua. Study on influential factors and countermeasures of farmers' income in Heilongjiang [M]. Harbin: Northeast Agricultural University, 2013. 
[19] Cao Yukun, Chen Jiancheng, Chen Lirong. Game analysis on subject behaviors and policies in state-owned forest tenure reform in Yichun [J]. Issues in Agricultural Economy, 2010(5):89-95 